\title{
MUSCULATURA DE LOS ARCOS BRANQUIALES, ARCO HIOIDEO Y OPERCULO EN PECES SCIAENIDAE DEL ATLANTICO OESTE Y RIOS DE AMERICA
}

Orangel Aguilera

\section{RESUMO}

A musculatura que serve aos arcos branquiais, arco hióideo e opérculc nos 22 gêneros marinhos do Atlântico oeste e nos 4 gêneros dos rios da Amé rica se caracterizam por apresentar a origem, percurso e inserção das seções e subseções musculares de maneira geral comum para todos os Sciaenidae estudados. Porém, existem algumas variações relacionadas principalmente com o grau de desenvolvimento desta musculatura, que para efeitos da descrição permite o agrupamento dos diferentes gêneros em três (3) grupos, o grupo "Macrodon" (Macrodon, Cynoscion, Larimus, Nebris, Isopistus, Plagioscion, Lonchurus, Stellifer, Odontoscion, Bairdiella e Corvulla), grupo "Micropogonias" (Micropogonias, Umbrina, Equetus, Pareques, Leiostomus, Sciaena, Ctenosciaena, Pachyurus, Pachypops, Menticirrhus, Paralonchurus $e$ Ophioscion) $e$ grupo "Pogonias" (Pogonias e Aplodinotus), mas este último se afasta do padrão geral devido à fusão dos quintos ceratobranquiais e ao desenvolvimento de um processo ventral no mesmo que determina algumas variações críticas a respeito da origem $e$ inserção dos músculos que o servem.

\section{INTRODUCCION}

Los estudios del sistema muscular que sirve a los arcos branquiales, arco hioideo y opérculo han .cobrado recientemente gran interés como lo demuestran los trabajos publicados por Liem $(1973 ; 1978 ; 1979 ; 1980)$ y Osse (1969), los cuales abordan aspectos funcionales, mientras que Winterbottom (1974) realiza estudios filogenéticos, y adicionalmente Liem (1981), y Liem \& Greenwood (1981), efectúan estudios combinados (funcionales y filogenéticos). No obstante, aunque el tema principal de esos trabajos no es la descripción anatómica, los mismos permiten ver la necesidad de adquirir un conocimiento de la anatomía básica para poder interpretar adecuada- 
mente aspectos funcionales y filogenéticos que puedan plantearse en el estudio de los Sciaenidae.

Entre los estudios más importantes de la musculatura estriada en Sciaenidae resalta el de Liem \&Greenwood (1981) quienes describen aspectos funcionales de los músculos que sirven a los arcos branquiales de Pogonias cromis; el de Jinadasa (1978) quien describe la musculatura facial y del arco hioideo en Paralonchurus y Lonchurus, y el de Aquilera (en prensa) quien describe la musculatura facial de Sciaenidae del Atlántico Oeste y de los ríos Americanos.

La finalidad del presente trabajo es describir la anatomía básica de la musculatura que sirve a los arcos branquiales, arco hioideo y opérculo de los 22 géneros marinos del Atlántico Oeste y en los 4 géneros de los ríos de América, para determinar las características miológicas de la familia.

\section{MATERIALES Y METODOS}

Se estudiaron 135 ejemplares, como se especifica en la lista de material examinado. Las tallas de los especímenes examinados oscilaron entre $53 \mathrm{~mm}$ $-356 \mathrm{~mm}$ LS.

En la nomenclatura utilizada para los músculos se siguió a Winterbottom (1974); en la. osteológica a Cervigón (1980). Los especímenes previamente fijados en formalina al $10 \%$ y conservados en alcohol isopropílico al $50 \%$ fueron disecados bajo observación en lupa estereoscópica. Los dibujos fueron realizados directamente con ayuda de una cámara clara. Por convención el término origen de un músculo se refiere a su unión con una estructura más o menos fija, mientras inserción cuando éste se une a una estructura móvil. Los músculos están representados en los dibujos por rayados de líneas contínuas, los ligamentos por pequeños trazos y los huesos en blanco. La descripción general de la musculatura se hizo basada en Micropogonias (Micropogonias furnieri) por presentar las características más frecuentes entre los Sciaenidae, así como una amplia distribución en el Atlántico Oeste.

Para facilitar la descripción de la musculatura que sirve a los arcos branquiales de los diferentes géneros, se agruparon por sus similitudes morfológicas en tres grupos distintos, tomándose como representante de cada grupo al género que define mejor sus características. En el caso de los músculos que sirven al arco hioideo y al opérculo, básicamente presentan una disposición invariable por lo cual su descripción se limita a la descripción general.

A continuación se presenta la lista del material examinado en orden alfabético. El número entre parántesis corresponde a los especímenes examinados:

Aplodinotus grunniens (1), 185 mm LS, 19: VI: 82', Río MissisippiSavana (U.S.A.). Bairdiella ronchus (5), $95-142 \mathrm{~mm} \mathrm{LS,} \mathrm{10:} \mathrm{VII:} \mathrm{80',} \mathrm{Chi-}$ 
guana-Golfo de Cariaco (Venezuela); (3), 137-156 mm LS, 10: III: 81', Laguna de la Restinga-Isla de Margarita (Venezuela); (2), 85-94 mm LS, 23: 11: 81', Río Sergipe-Aracajú (Brasil) (2), 140-152 mm LS, 28: III: 80', Tuxpan-Veracrúz (México). Bairrdiella chrysoura (2), 68-75 mm LS, 25: VII: 81', Sontecomapun-Verecruz (México). Corvula sanctaeluciae (5), 112-134 mm LS, 10: VII: 80', Playa El pefión-Golfo de Cariaco (Venezuela). Ctenosciaena gracilicirrhus (3), 143-149 mm LS, 3: V: 81', Bahía de Móchima (Venezuela); (4), 118-143 mm LS. 10: III: 78', Farol Sarita-Río Grande do Sul (Brasil); (1) mm LS, 1:82', 3052' S y $50^{\circ}$ W (Brasil). (1), 142 mm LS, I: $82^{\prime}, 30^{\circ} 52^{\prime} \mathrm{S}$ y $50^{\circ} 10^{\prime} \mathrm{W}$ (Brasil). Cynoscion similis (5), 194-223 mm LS, 8: VIII: 81., Bahía de Mochima (Venezuela). Cynoscion striatus (2), 63-84 mm LS, 28: X: 80'. Praia do Cassino-Río Grande do Sul (Brasil). Cynoscion microlepidotus (2), 114-125 mm LS, X: 80', Río Sergipe-Aracajú (Brasil). Cynoscion jamaincensis (3), 207-216 mm LS, I: 82', 3851'S $50^{\circ} 19^{\prime} \mathrm{W}$ (Argentina). Equetus lanceolatus (1), 147-153 mm LS, 3: V: 81', Bahía de Mochima (Venezuela). Larimus brevisceps (4), 154-187 mm LS, 19: VIII: 80', Golfo de Cariaco-Cumaná (Venezuela); (2), 78-85 mm LS, 23: II: 81' Río Sergipe-Aracajú (Brasil). Leoistomus xanthurus (2), 96-119 mm LS, 7: VII: 63', Isle of Wight Bay-Maryland (U.S.A.). Lonchurus lanceolatus (1), $108 \mathrm{~mm}$ III: 81', Delta del Río Orinoco (Venezuela). Micropogonias furnieri (6), 182-195 mm LS, 8: VIII: 81', Bahía de Mochima (Venezuela); (2), 165-180 mm LS, 10: VII: 80, Golfo de Cariaco (Venezuela); (4), 80-146 mm LS, 3: 1:81', Lagoa dos Patos-Río Grande (Brasil). Micropogonias undulatus (1), 236 mm LS, 4: VI: 76', Río Missisippi (U.S.A.); (1), 73 mm LS, 22: VIII: 80', Mandinga-Veracrúz (México). Macrodon ancylodon (6), 80135 mm LS, 20: XI: 80', Praia do Cassino-Río Grande (Brasil). Menticirrhus americanus (5), 73-125 mm LS, 17: II: 81', Lagoa dos Patos-Río Grande (Brasil). Menticirrhus littoralis (4), 62-187 mm LS, 11 : III: 81', Playa La Galera-Isla de Margarita (Venezuela); (3), 80-114 mm LS, 25: IV: 80', Farol Sarita-Río Grande do Sul (Brasil). Menticirrhus saxatilis (1), 125 mm LS, 7: IX: 66', Suffolk Co New York (U.S.A.). Nebris microps (1), $216 \mathrm{~mm} \mathrm{LS}$, donado el 8: XI: 81', (sin fecha colecta), Golfo de PariaPuerto de Guiria (Venezuela). O dontoscion dentex (4), 142-151 mm LS, 19: VI: 81 ', Playa de Turpialito (Venezuela). O phioscion punctatissimus (1), 157 mm LS, 11: VII: 72', Playa San Luis-Cumaná (Venezuela); (3), 88-157 mm LS, 23: II: 81', Río Sergipe-Aracajú (Brasil). Paralonchurus brasiliensis (6), 112-170 mm LS, 23: VI: 80', Sao José do Norte-Río Grande do Sul (Brasil). Pachypops fourcroi (1), 91 mm LS, 30: V: 81', Río OrinocoTucupita (Venezuela). Pachypops adspersus (2), 180-187 mm LS, X: II: 78', Lagoa Feia-Río Janei ro (Brasil) Pachyurus scomburgkii (2), 80-95 mm LS, X: 79', Río Negro Amazonas (Brasil). Pachypops trifilis (2), 125-132 mm LS, 22: IV: 79', Río Solimoe-Coarí (Brasil). Pareques acuminatus (3), 158-162 mm LS, 18: XII: 80', Bahía de Mochima (Venezuela). Plagioscion squamusissimus (5), 236-259 mm LS, 16: X: 81', Río Orinoco-Tucupita (Venezuela); (2), 67-88 mm LS, 23: 1: 79', Janavaca-Amazonas (Brasil). Pogonias cromis 
(6), 85-1 56 mm LS, 28: X: 80', Lagoa dos Patos-Rio Grande (Brasil). Sciaena bathytatos (4), 254-356, mm LS, 15: IX: 80', Cañón Sub-marino del Río Manzanares - Cumaná (Venezuela). Sciaenops ocellata (1), $118 \mathrm{~mm}$ LS, 12: V: 49', Patuscent-Maryland (U.S.A.). Stellifer venezuelae (3), 53-78 mm LS, 18: VII: 69', laguna de Tacarigua-Edo. Miranda (Venezuela). Stellifer stellifer (2), 87-1 15 mm LS, X: 80', Río Sergipe-Aracajú (Brasil). Stellifer brasiliensis (2), 95-103 mm LS, X: 80', Río Sergipe-Aracajú (Brasil). Stellifer rastrifer (2), 85-122 mm LS, 23: 11:81', Río Sergipe-Aracajú (Brasil), Umbrina coroides (4), 142-165 mm LS, 10: VII: 80', Golfo de CariacoCumaná (Venezuela); (3), 52-82 mm LS, 11: II: 81', Playa La Galera-Isla de Margarita (Venezuela). Umbrina canossai (6), 92-122 mm LS, 17: VI: 80', Lagoa dos Patos-Río Grande (Brasil): (2), 142-156 mm LS, I: 82', 3354' S - 52041'W (Uruguay).

\section{RESULTADOS}

Características de la musculatura que sirve a los Arcos Branquiales, Arco Hioideo y Opérculo en Sciaenidae.

El origen, recorrido e inserción de los músculos que sirven a los arcos branquiales, arco hioideo y opérculo es común para todos los Sciaenidae examinados. La musculatura se describe sobre la base del género Micropogonias (Micropogonias furnieri), por presentar las características más frecuentes entre los Sciaenidae, aunque los diferentes géneros muestran algunas variaciones relacionadas principalmente con el grado de desarrollo de las secciones musculares.

Músculos que sirven a la parte dorsal de los arcos branquiales.

Levatores externi (L. ext. Fig. 1A-B): Unen a los epibranquiales con el neurocráneo. Se originan en la superficie ventral del pterótico y extremo anterodorsal del proótico, y se insertan dorsolateralmente a los cuatro epibranquiales.

Levatores interni (L. int. Fig. 1A-B): Unen a los primeros faringobranquiales con el neurocráneo. Son internos a los levatores externi y se originan en el proótico, para insertarse en el segundo y tercer faringobranquial.

Levator posterior (L. post. Fig. 1A-B): se originan en la superficie ventral del pterótico para insertarse en el cuarto epibranquial, justamente posterior y externamente a la inserción del L. ext. IV.

Obliqui dorsales (Obl.d.Fig. 1B): Estos músculos unen a los epibranquiales con sus respectivos faringobranquiales. En Sciaenidae encontramos el Obl.d.posterior (Obl.d. III y IV) que se encuentra formado por fibras anteriores que se originan en el tercer epibranquial y otras posteriores que se originan en el cuarto epibranquial, para insertarse al tercer faringobranquial. 


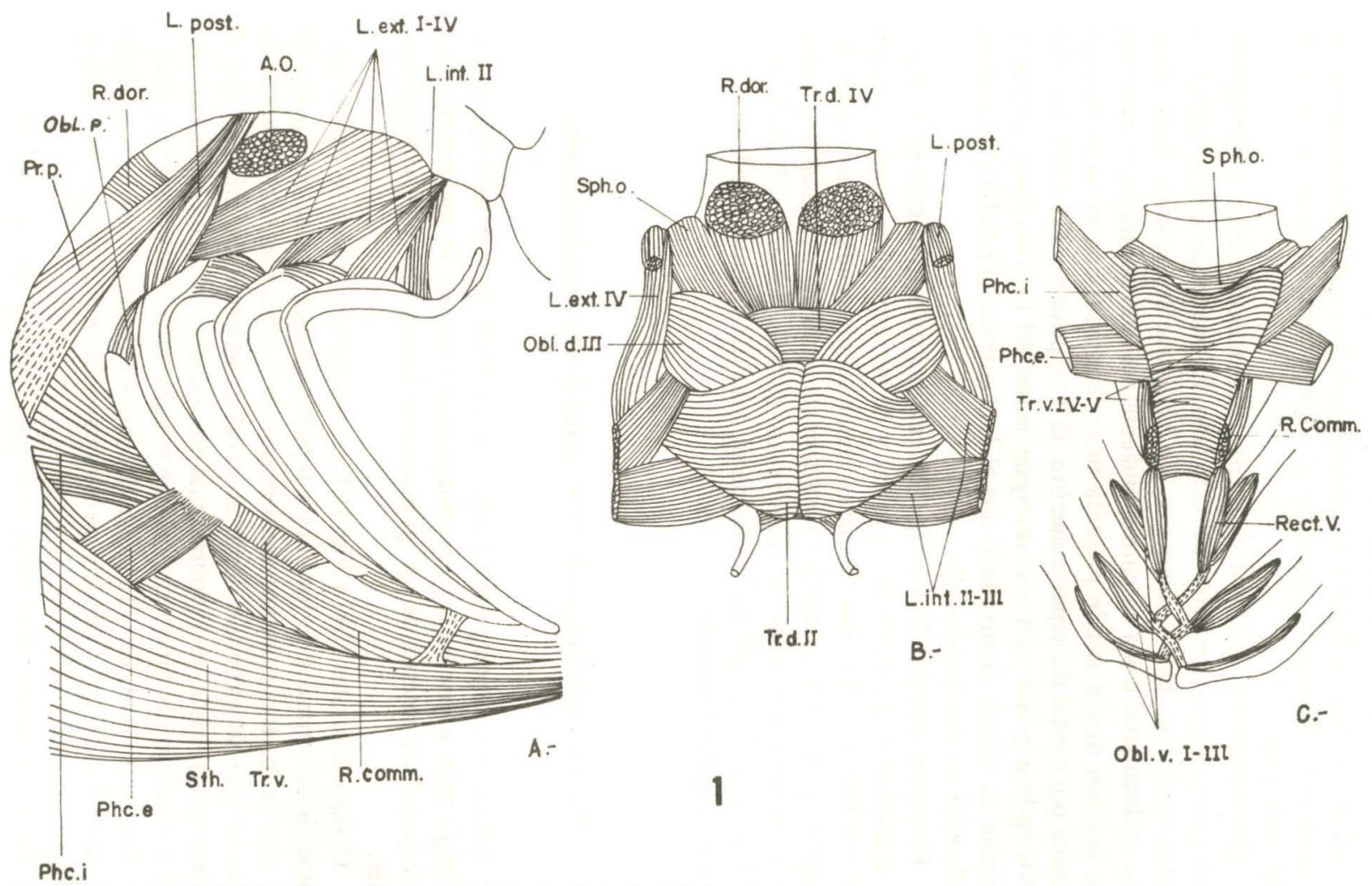

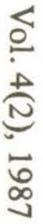

Fig. 1. Musculatura branquial de Micropogonias furnieri (A) vista lateral, (B) vista dorsal y (C) vista ventral. 
Obliquus posterior (Obl.p.Fig. 1A): este músculo une el extremo posterior del quinto ceratobranquial con la superficie posterointerna del cuarto ceratobranquial y cuarto epibranquial.

Adductor (Ad. s/ Fig.): este músculo une al cuarto epibranquial con su respectivo ceratobranquial, próximo al ángulo formado entre la unión de ambos elementos.

Transversi dorsales (Tr. d. Fig. 1B): estos músculos unen a los epibranquiales con su correspondiente del otro lado. En Sciaenidae encontramos dos de estos músculos. El Tr. d.anterior (Tr.d.III) que se extiende desde el segundo epibranquial hasta su correspondiente del lado contrario, se ensancha en su porción media donde presenta fuerte contacto con el tercer faringobranquial por medio del tejido conectivo. El Tr.d.posterior (Tr.d.IV) une a los cuartos epibranquiales con sus car respondientes del lado contrario, comparativamente es menos desarrollado que el Tr.d.anterior (Tr.d.III) y se dispone dorsalmente al retractor dorsalis.

Retractor dorsalis (R.dor.Fig. 1B): este músculo se origina en la superficie laterodorsal del centrum vertebral y de las respectivas espinas neurales correspondientes a las tres primeras vértebras pre-caudales; así como del exocipital, para luego converger medialmente (uno de cada lado), pasando entre la superficie ventral del transversus dorsalis posterior (Tr.d.IV) y la superficie dorsal del sphincter oesophagi, para finalmente insertarse en los terceros y cuartos faringobranquiales.

\section{Músculos que sirven a la parte ventral de los arcos branquiales}

Sphincter oesophagi (Sph.o.Fig. 1C): las fibras de este músculo rodean al esófago, para unirse en la línea media dorsal y ventral. Se une anteriormente a la superficie interna de los quintos ceratobranquiales y cuartos epibranquiales.

Obliqui ventrales (Obl.v.Fig. 1C): estos músculos sirven de unión entre las superficies ventrales de los huesos hipobranquiales y ceratobranquiales de cada uno de los tres primeros arcos branquiales, estos a su vez presentan un ligamento que se extiende desde el extremo anterior del músculo (en el hipobranquial) hasta el hipobranquial precedente del lado contrario; con excepción del primer hipobranquial que se une a su respectivo basibranquial.

Transversi ventrales (Tr.v.Fig. 1C): estos músculos unen las superficies ventromediales de los cuartos y quintos pares de ceratobranquiales respectivamente.

Rectus ventralis (Rec.v.Fig. 1C): este músculo une la superficie ventrointerna del extremo inferior del cuarto ceratobranquial con el proceso ventral del tercer hipobranquial por medio de un ligamento.

Rectus communis (R.comm.Fig. 1C): este músculo une el extremo anterior de los quintos ceratobranquiales con la parte superior del urohial. 
Músculos entre la cintura pectoral, el craneo, arco hioideo y el arco branquial

Sternohyoideus (Sth.Fig. 1A): este músculo se origina en el cleitrum y cor acoides, sus fibras posterolaterales se unen a la superficie del miocoma que lo separa de la porción anterior del músculo obliquus inferioris (Hypaxialis) y para su inserción ambos músculos convergen medialmente a cada lado del urohial hasta los hipohiales a los cuales se une por medio de ligamentos. En la región de convergencia se desarrolla una aponeurosis que se une al proceso ventral de los terceros hipobranquiales.

Pharyngoclavicularis externus (Phc.e.Fig. 1Ay C): se origina en la región más distal del cleitrum (pasando entre las fibras del sternohyoideus), y se inserta en la superficie lateroventral del extremo anterior de los quintos ceratobranquiales.

Pharygoclavicularis internus (Phc.i.Fig. 1A y C): se origina en la región anterior del cleitrum, dorsalmente al origen del Phc.e. y se inserta en la superficie ventrointerna de los quintos ceratobranquiales.

Protractor pectoralis (Pr.p.Fig. 1A): se origina en el pterótico y se inserta en la superficie anterodorsal del cleitrum.

Levator pectoralis (L.pect.Fig. 1A): este músculo se origina en el pterótico y se inserta en el postemporal y supracleitrum.

\section{Músculos de la superficie ventral de la cabeza}

Intermandibulares (Imd.Fig. 2): este músculo se localiza en la sínfisis de la mandibula inferior, extendiéndose transversalmente entre ambos dentarios a través de las fibras del protractor hyoidei.

Protractor hyoidei (Pr.hi.Fig. 2): éste músculo une al arco hyoideo con la mandibula inferior. Se origina en la superficie interna del dentario próximo a la sínfisis, posteriormente se une al cerotohial y a los primeros radios branquiostegos.

Hyohyoideo abductores (H.ab.Fig. 2): éstos músculos se originan en los hipobranquiales ventrales, para insertarse en el primer radio branquiostego del arco hyoideo contrario. No obstante, fibras secundarias de este músculo sirven tambien a los segundos y terceros radios branquiostegos.

Hyohyoideo adductores (H.ad.Fig. 2): éstos músculos se extienden entre los radios branquiostegos, siendo que las fibras más posteriores se unen a la superficie interna del opérculo y sub-opérculo.

\section{Músculos entre el neurocraneo y el opérculo}

Dilator operculi (D.o.Fig. 3): éste músculo une la región posterorbital del neurocráneo con el opérculo. Anteriormente se encuentra cubierto 
por el levator arcus palatini y el extremo dorsal del preopérculo. Se origina en el esfenótico y pterótico, en la fosa dilatora y en la superficie externa del hiomandibular, dorsalmente al eje formado entre el cóndilo anterior y el posterior, para insertarse en la superficie anterodorsal del opérculo.

Levator operculi (L.op.Fig. 3): se origina del borde posterior del hiomandibular, dorsalmente al cóndilo posterior y se inserta a la superficie dorsamedial del opérculo. Es posterior al dilator operculi y sus fibras anteriores están en contacto con éste.

Adductor operculi (A.o.Fig. 3): se extiende desde la pared lateral de la región auditiva del neurocráneo hasta la superficie dorsointerna del opérculo. Se origina en la superficie ventral del pterótico y extremo posterodorsal del proótico para insertarse en la superficie interna del opérculo, justamente posterior a la faceta articular.

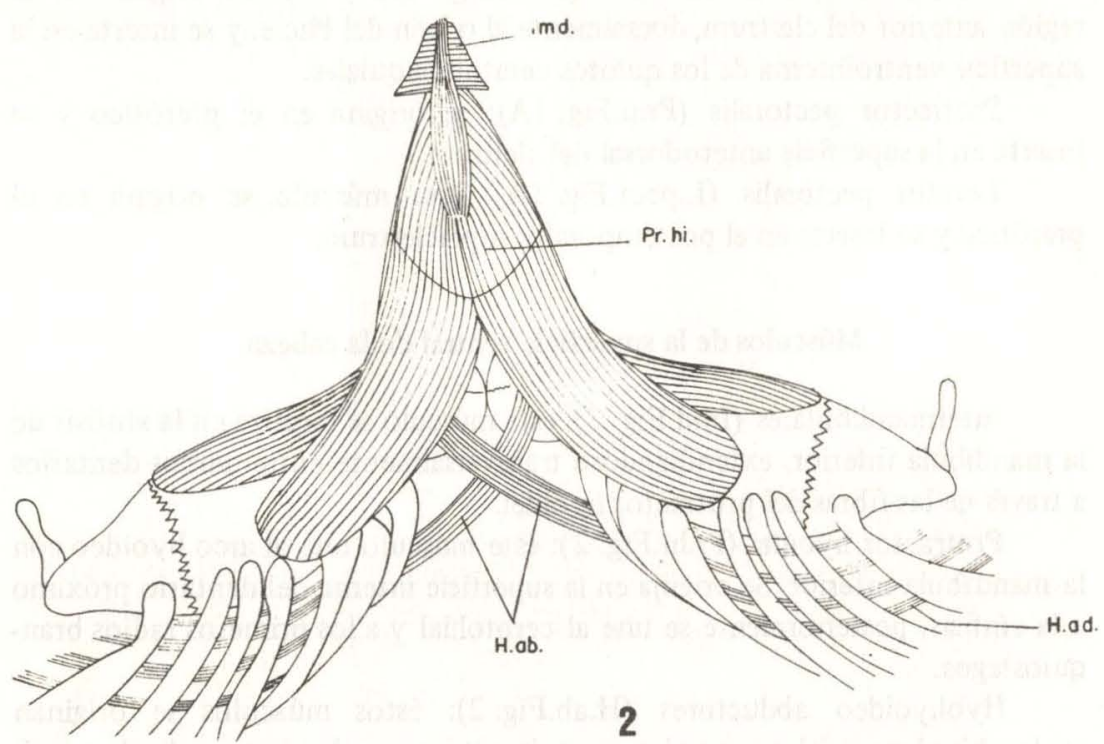

Fig. 2. Vista ventral de la musculatura del arco hioideo de Micropogonias furmieri.

\section{DESCRIPCION GENERAL DE LA MUSCULATURA DE LOS ARCOS BRANQUIALES, ARCO HIOIDEO Y OPÉRCULO EN LOS DISTINTOS GRUPOS GENEERICOS}

Para facilitar la descripción de ésta musculatura en los diferentes géneros, fueron agrupados por su similitud morfológica en tres (3) grupos, to- 


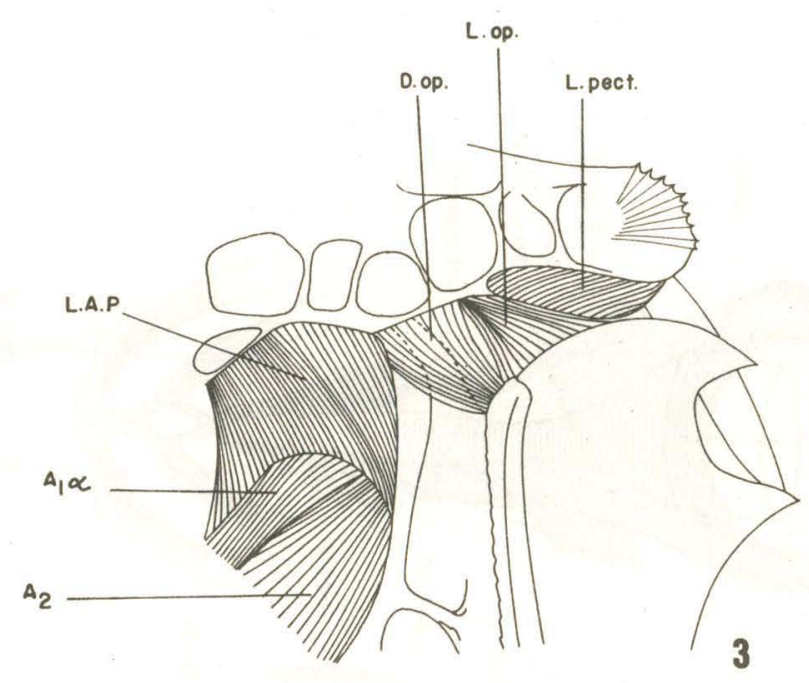

Fig. 3. Vista lateral de la musculatura del opérculo de Micropogonias furnieri.

mando como representante de cada grupo el género que define mejor las características del grupo. En caso de los músculos que sirven al arco hioideo y al opérculo, por presentar una disposición invariable, su descripción se limita a la descripción general anterior.

Grupo "Macrodon" (Fig. 4): Plagioscion, Macrodon, Cynoscion, Larimus, Sciaenops, Nebris, Isopisthus, Lonchurus, Stellifer, Odontoscion, Bairdiella y Corvula, integran este grupo, que se caracteriza por presentar los músculos que sirven a la parte dorsal de los arcos branquiales largos, delgados y estrechos, presentando de manera general cierta porporción entre ellos. Por otro lado, los músculos que sirven a la parte ventral de los arcos branquiales y entre éstos y la cintura pectoral, a pesar de ser largos y delgados, en general son muy anchos, como por ejemplo el pharingoclaviculariş externus. Esta disposición aparentemente se encuentra relacionada con la mayor longitud de los arcos branquiales y del urohial, así como el escaso desarrollo de los dientes faríngeos, lo cual probablemente indica que la función principal de esta musculatura sea la de promover el movimiento que conduzca el alimento directamente al esófago, sin que halla una trituración previa de éste. En los géneros Bairdiella, Corvula, Stellifer y Odontosción, los músculos levator posterior y protractor pectoralis se encuentran aparentamente menos desarrollados, probablemente por la presencia de los "cuernos" de la cámara anterior de la vejiga gáseosa.

Grupo "Micropogonias". (Fig. 1): Micropogonias, Umbrina, Equetus, Pareques, Leiostomus, Sciaena, Ctenosciaena, Pachyurus, Pachypops, Menticirrhus, Paralonchurus y Ophioscion integran este grupo, el cual se caracteriza por presentar la musculatura branquial comparativamente más desarrolla- 


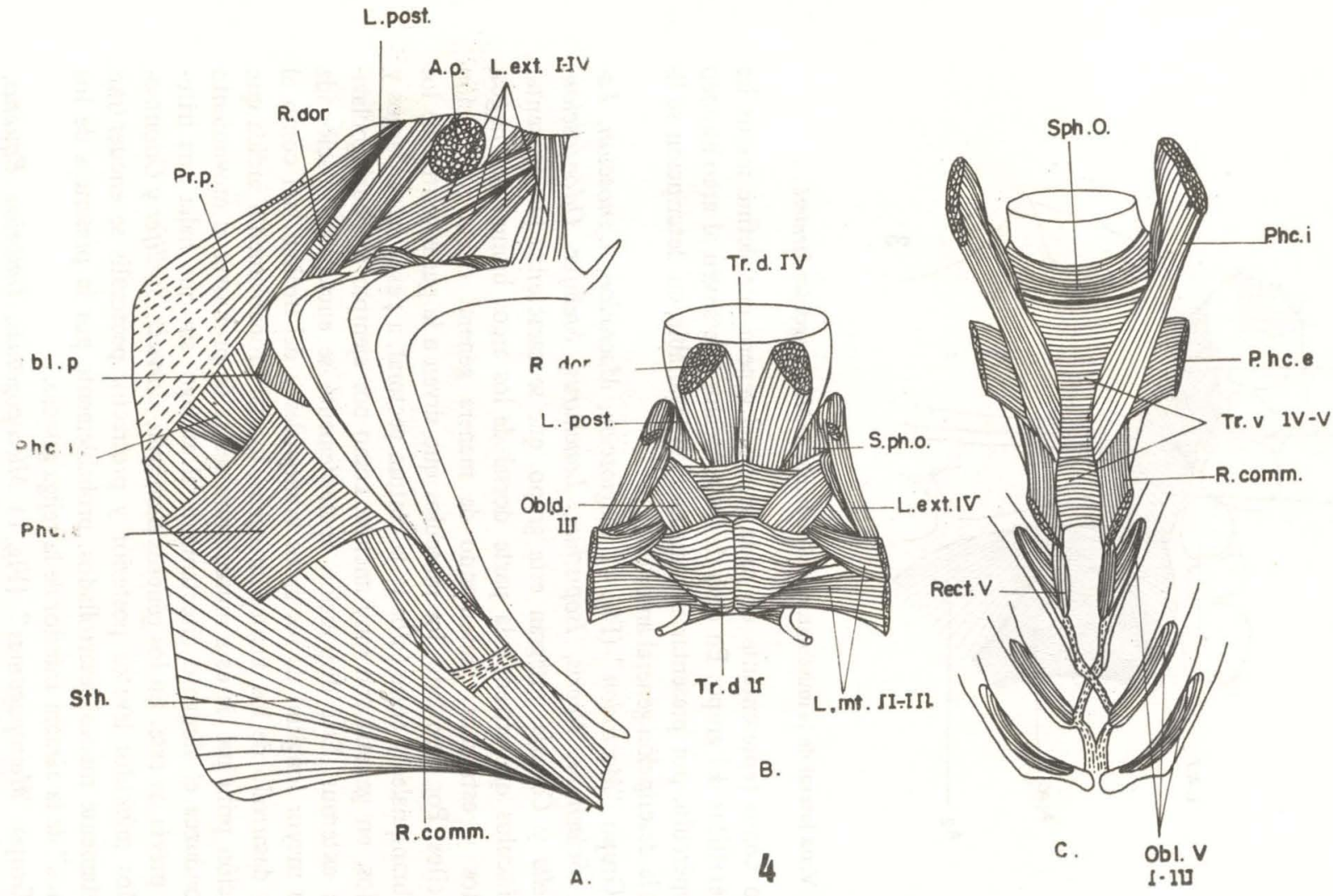

Fig. 4. Musculatura branquial de Macrodon ancylocion: A) vista tateral, (B) vista dorsal $y(C)$ vista ventral. 
da que el grupo anterior. En general los músculos son cortos y voluminosos, existiendo una mayor desproporción entre las secciones musculares, como es el caso del levator ex ternus IV, por ser el más voluminoso entre los levatores externi. Esta disposición probablemente se encuentra relacionada con la menor longitud de los arcos branquiales y dél urohial, así como el mayor grado de desarrollo de los dientes faríngeos, lo cual probablemente indica que estas especies puedan realizar una trituración previa del alimento capturado. En el caso de Ophioscion los músculos levator posterior y protractor pectoralis son comparativamente menos desarrollados, probablemente a consecuencia de la cámara anterior de la vejiga gaseosa.

Grupo "Pogonias". (Fig. 5): los géneros Pogonias y Aplodinotus integran este grupo, los cuales presentan en común la fusion de los quintos ceratobranquiales por medio de una sinartrosis, así como el desarrollo de un nuevo proceso ventral en el mismo, al cual se insertan los músculos pharingoclavicularis externus e internus, rectus communis y esternohyoideus. No obstante $\boldsymbol{P}$. cromis presenta un mayor desarrollo de las placas o dientes faríngeos superiores e inferiores, y consecuentemente ocurre una hipertrofia de la musculatura, al mismo tiempo que se desarrolla una apófisis faríngea en forma de ancla en la base del cráneo, al margen del cual se unen todos los levatores que sirven a la parte dorsal de los arcos branquiales. Esta disposición probablemente se encuentra relacionada con los hábitos alimentarios de ambos integrantes del grupo, debido al desarrollo de los dientes faríngeos y consecuentemente de su musculatura como para poder triturar lo que debe constituir su alimento principal.

\section{DISCUSION}

En general, desde el punto de vista anatómico, la musculatura estriada que sirve a los arcos branquiales, arco hioideo y opérculo en Sciaenidae se asemeja a la más frecuente descrita para Teleostei por Winterbottom (1974 a.b.), no obstante existen algunas variaciones como es el caso del número de obliqui dorsales que frecuentemente es de dos en Teleostei y aún cuando muchos Acanthopterygii solo presentan el obliques dorsales anterior, en Sciaenidae solo encontramos el obliques dorsales posterior (Obl.d.III + IV). Así mismo Dietz (1914), señala que el levator externi III generalmente se encuentra ausente en Acanthopterygii especializados, no obstante Liem \& Greenwood (1981) indican no solo su presencia, sino tambien su gran desarrollo en Pharyngognathi (grupo que presenta en común los 5o ceratobranquiales funsionados y en el cual se incluye Pogonias cromis), así mismo todos los Sciaenidae estudiados presentan el levator externi III.

Desde el punto de vista funcional se puede notar que existe una relación entre el desarrollo de la musculatura branquial y el de las placas ó dientes faríngeos, lo cual está aparentemente condicionado por los hábitos alimenticios de las especies en cuestión. 


\section{POS1}

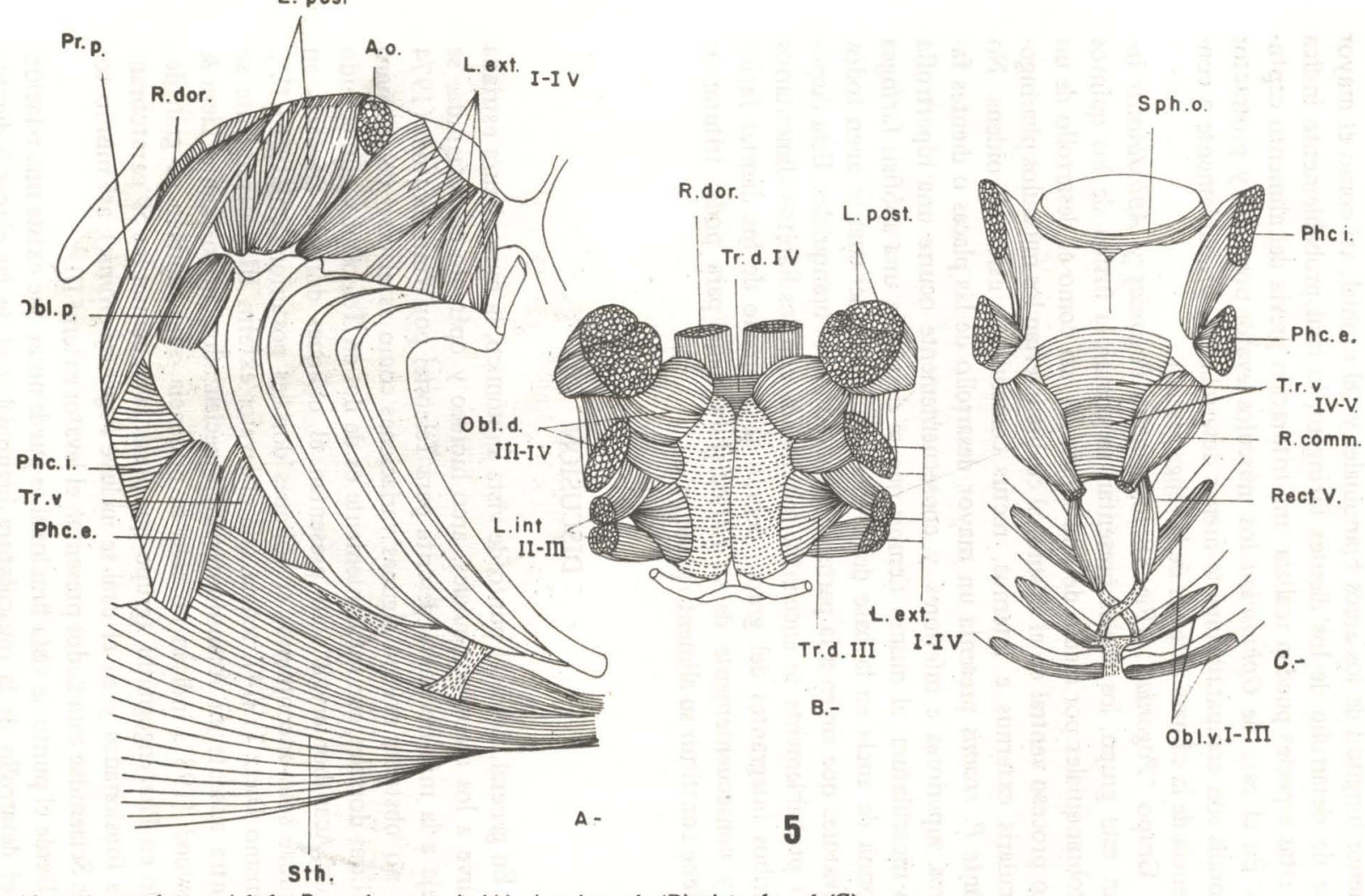

F1g. 5. Musculatura branquial de Pogonias cromis (A) vista lateral, (B) vista dorsal (C) ista ventral. 
Por otro lado, la musculatura estriada estudiada no obedece a un patrón específico que permita establecer grupos de referencia a no ser para facilitar la descripción, como es el caso de los músculos que sirve al arco hioideo y opérculo, los cuales básicamente presentan una disposición invariable en la familia. En el caso de los músculos que sirven a los arcos branquiales se observaron algunas características que permiten agrupar los Sciaenidae estudiados en tres (3) grupos, pero para efectos de variación significativa desde el punto de vista anatómico y funcional, solo el grupo "Pogonias" se aparta del patrón general debido a la fusión de los quintos ceratobranquiales, así como por el desarrollo de un proceso ventral que lógicamente determina algunas variaciones críticas con respecto al origen e inserción de los músculos que lo sirven.

Al contrario de los otros géneros de esta familia que no presentan una unión basifaríngea con las placas faríngeas superiores, en $P$. cromis y $L$. xanthurus existe una apófisis faríngea similar a la que señala Greenwood (1965) para peces malacófagos y Liem \& Greenwood (1981) para Pharyngognathi. .Sin embargo, aún cuando $L$. xanthurus presenta esta apófisis, no ocurre fusión de los quintos ceratobranquiales, ni se desarrolla un proceso ventral en el mismo, como es el caso del grupo "Pogonias", no obstante presenta un buen desarrollo de la musculatura que sirve a los arcos branquiales y los dientes de la placa faríngea son del tipo molariformes.

Por otra parte resulta interesante sefialar que en $P$. cromis y $A$. grunnies aparte de los músculos pharingoclavicularis externus e internus, y rectus communis, el músculo sternohyoideo tambien se inserta al proceso ventral de los quintos ceratobranquiales, lo cual aparentemente le confiere propiedades biomecánicas diferentes relacionadas con el manipuleo y preparación del alimento, cuando se les compara con los otros géneros integrantes de esta familia. Sin embargo, esta manipulación deberá ser definitivamente mayor en $P$. cromis que en $A$. grunniens dado a que $P$. cromis presenta una apófisis basifaríngea bien desarrollada, así como las placas faríngeas y los músculos que la sirven.

Opuesto a lo observado con la musculatura del grupo "Pogonias", en el grupo "Micropogonias" y especificamente en $\boldsymbol{M}$. furnieri (quien presenta lo que hemos considerado la forma más frecuente de esta musculatura), resulta interesante observar la aparente versatilidad de la misma, lo cual le permitirá explorar una mayor diversidad de los recursos alimenticios en los diferentes niveles de la columna de agua y del fondo.

Finalmente, se observa de manera general la constancia en cuanto al origen, recorrido e inserción de las secciones y subsecciones musculares en esta familia, tal como lo describe Aguilera (en prensa) sobre la base de la musculatura facial.

\section{AGRADECIMIENTOS}

Le expreso mi agradecimiento al Dr. Labbish Chao (Universidade 
de Río Grande, Brasil), por sus valiosas críticas, donación de material ictiológico, y por sus facilidades de laboratorio. Tambien a las siguientes personas e instituciones por la donación y/o el préstamo de ejemplares: Dr. S. Stewart (Field Museum of Natural History), Dr. R. Robins (Rosenstiel School of Marine and Atmospheric Science), Dr. J. Musick (Virginia Institute of Marine Science), Dr. G. Nelson (American Museum of Natural History), Prof. J. Martínez (Escuela Nacional de Estudios Profesionales Iztacala de México), Profesores: S. Anibal y G. Mendez dos Santos (Instituto Nacional de Pesquisas da Amazônia, Brasil). Así mismo, por las oportunas sugestiones y críticas al Dr. F. Mago-Leccia (Universidad Central de Venezuela).

\section{BIBLIOGRAFIA}

Aquilera, O. Én prensa. Musculatura facial de peces escienidos (Perciformes: Sciaenidae) del Atlantico Oeste y ríos de America. Mem. Fund. La Salle de Ciencias Naturales Vol.119.

Dietz, P.A. 1914. Beitrage zur Kenntnis der Kiefer und Kiemenbogenmuskulatur der Teleostier, I. Die Kieferund Kiemenbogenmuskeln der Acanthopterygii. Mitteilungen Stazione Zoologica Neapel, 22 (4): 99-162.,

Greenwood, P.H. 1965. Environmental effects on the pharyngeal mill of a cichlid fish, Astatoreochromis alluaodi, and their taxonomics implications. Proc. Limm. Soc. Lond. 176: 1-10.

Jinadasa, J. 1978. Biology of the sciaenid genera Paralonchurus and Lonchurus (Pisces, Sciaenidae). Univ. Mas, Ph D.Diss, 261 p.

Liem, K.F. 1973. Evolutionary strategies and morphological innovations Cichlid pharyngeal. - Syst. Zool., 22 (4): 425-441.

Liem, K.F. 1978. Modulatory multiplicity in the funcional repertoire of the feeding mechanisms in cichlid Fishes. - J. Morph. 158 (3): 323-360.

Liem, K.F. 1979. Modulatory multiplicity in the feeding mechanisms in cichlid fishes, as exemplified by the invertebrate pickers of lake Tanganyika. - J. Zool, 189: 93-125.

Liem, K.F. 1980. Acquisition of energy by Teleosts: Adaptive mechanisms and evolutionary patterns. - Environmental Physiology of fishes. M.A. Ali. (Ed.)., 299-334.

Liem, K.F. 1981. A phyletic study of the lake Tanganyika cichlid genera Asprotilapia, Ectodus, Lestradea, Cunningtonia, Ophthalmotilapia, and Ophthalmochromis. Bull. Mus. Comp. Zool. 149 (3): 191-214.

Liem, K.F. \& P.H. Greenwood 1981. A funtional approach to the phylogeny of the pharyngognath teleosts. - Am. Zool. 21: 83-101.

Osse, J.W.M. 1969. Functional morphology of the head of the perch (Perca fluviatilis L.) : An electromyographie study. - Netherlands J. Zool. 19 (3): 289-392.

Winterbottom, R. 1974 a. A descriptive synonymy of the striated muscles of the Teleostei. - Proc. Acad. Nat. Sci. Phi., 125 (12): 225-317.

Winterbottom, R. 1974 b, The familial phylogeny of the Tetraodontiformes (Acanthopterygii, Pisces), as evidenced by their conparative myology. - Smithsonian Contr. Zool., 201p. 
Músculos que sirven a la parte dorsal de los arcos branquiales:

$\begin{array}{ll}\text { Levatores extemi } & \text { L. ext. } \\ \text { Levatores interni } & \text { L. int. } \\ \text { Levator posterior } & \text { L. post. } \\ \text { Obliqui dorsales } & \text { Obl.d. } \\ \text { Obliquus posterior } & \text { Obl.p. } \\ \text { Transversi dorsales } & \text { Tr.d. } \\ \text { Retractor dorsalis } & \text { R.dor. }\end{array}$

Músculos que sirven a la.parte ventral de los arcos branquiales:

$\begin{array}{ll}\text { Sphincter oesophagi } & \text { Sph.o. } \\ \text { Obliqui ventrales } & \text { Obl.v. } \\ \text { Transversi ventrales } & \text { Tr.v. } \\ \text { Rectus ventralis } & \text { Rec.v. } \\ \text { Rectus communis } & \text { R.comm. }\end{array}$

Músculos entre la cintura pectoral, el cráneo, arco hioideo y el arco branquial:

Stemohyoideus

Pharyngoclavicularis externus

Pharyngoclavicularis internus

Protractor pectoralis

Leva tor pectoralis
Sth.

Phc.e.

Phc.i.

Pr.p.

L.pect.

Músculos de la superficie ventral de la cabeza:

$\begin{array}{ll}\text { Intermandibulares } & \text { Imd. } \\ \text { Protractor hyoidei } & \text { Pr hi. } \\ \text { Hyohyoideo obductores } & \text { H.ab. } \\ \text { Hyohyoideo adductores } & \text { H.ad. }\end{array}$

Músculos entre el neurocráneo y el opérculo:

Dilator

D.o.

Levator operculi

L.o.

Adductor operculi

A.o. 